\title{
ARYL HYDROCARBON RECEPTORS IN INDOLE DERIVATIVE TREATED MICE: NEUROPHARMACOLOGICAL PERSPECTIVES
}

\author{
P. Andreeva-Gateva', J. Tchekalarova², K. Kamenova', M. Strokova-Stoilova', S. Chakar', \\ R. Hadjiolova ${ }^{4}$, V. T. Angelova ${ }^{3}$, SI. Surcheva ${ }^{1}$ \\ 'Department of Pharmacology and Toxicology, Faculty of Medicine, Medical University - Sofia, Bulgaria \\ ${ }^{2}$ Institute of Neurobiology, Bulgarian Academy of Sciences \\ ${ }^{3}$ Department of Chemistry, Faculty of Pharmacy, Medical University - Sofia, Bulgaria \\ ${ }^{4}$ Department of Pathophysiology, Faculty of Medicine, Medical University - Sofia, Bulgaria
}

\begin{abstract}
Aim/objective. When applied in pharmacological doses, the indole derivative melatonin exhibits neuroactive and neuroprotective effects. Indoles and their metabolites, such as kynurenine, are ligands of aryl hydrocarbon receptors (AhR). This study aimed to evaluate the antiepileptic and analgesic activity of melatonin and two synthetic melatonin derivatives. The possible involvement of AhR and kynurenine in their neuropharmacological effects were also tested. Methods. The tested substances were: melatonin, two melatonin derivatives bearing aryl hydrocarbon moiety with either furyl or thienyl substitute (3e and 3f), and alpha naphthoflavone (ANF), an antagonist of AhR. After intraperitoneal injection of 30,100 , or $300 \mathrm{mg} / \mathrm{kg}$ of the tested agents for seven days, male mice ICR (25-30 g) were subjected to a corneal kindling seizure model. Two tests for analgesia, i.e., the hot plate test and the formalin test, were also applied. AhR and kynurenine concentrations were evaluated in brain homogenates. Results. Substances $3 e$ and $3 f$ demonstrated an antiepileptic activity comparable to that of melatonin. Some analgesic activity was also shown, albeit lower than that of melatonin in equivalent doses. For melatonin and $3 f$ treated mice, dose-dependent increases in AhR and kynurenine levels in brain homogenates were recorded. The antagonist ANF neither blocks the antiseizure activity of the tested indoles, nor demonstrated analgesic activity. Conclusion. Melatonin and the two tested melatonin-aroylhydrazone derivatives bearing either furyl or thienyl substitute exhibit antiepileptic and analgesic activity. Our results did not support the involvement of AhR in the demonstrated neurobiological activity. Further studies are needed to elucidate their exact molecular mechanisms.
\end{abstract}

Key words: melatonin, indole derivatives, corneal kindling, analgesia, aryl hydrocarbon receptors, kynurenine

Corresponding author: Pavlina Andreeva-Gateva, Department of Pharmacology and Toxicology, Medical Faculty, Medical University, 1, Georgi Sofiiski Street, 1431 Sofia, Bulgaria, E-mail: pandreeva_gateva@outlook.com,ORCID: 0000-0002-3791-8654

REVISED: 26 June 2020, ACCEPTED: 26 July 2020 


\section{INTRODUCTION}

T he aryl hydrocarbon receptor (AhR) is a highly conserved ligand-activated transcription factor of the basic helix-loop-helix Per-Arnt-Sim (PAS) family among vertebrates. Upon binding to its ligands, cytoplasmic AhR is transferred to the nucleus. After heterodimerization with aryl hydrocarbon receptor nuclear translocator (ARNT), it mediates numerous biological and toxicological effects by transcribing various AhR-responsive genes [1]. Well-known targets for AhR are CYP1A1, CYP1B1, ABCG2, ALDH3A1, NRF2, and UDP-glucuronosyltransferases (UGTAs) genes, and many other genes found to be either up- or down-regulated by AhR activation [2]. Further, AhR regulates a variety of biological processes involved in cell division, differentiation, apoptosis, sex hormone activity, reproduction, and immunity [3]. It was also shown that AhR possesses the intrinsic E3 ubiquitin ligase function that is important for proteasomal degradation [4].

AhR-ligands range from industrial substances, pharmaceuticals, or microbiome products, to natural compounds from plant origin [5]. The majority of high-affinity AhR-ligands are synthetic substances formed as a result of a non-biological activity. They are halogenated aromatic hydrocarbons and polycyclic aromatic hydrocarbons [6]. Natural sources of ligands, such as flavonoids, carotenoids, and glucobrassicin, are derived from fruits and vegetables [7].

Exogenous diet-derived indoles, such as tryptophan, constitute a significant source of AhR ligand precursors, produced via four pathways: i) hydroxylation to serotonin and melatonin; ii) decarboxylation to tryptamine; iii) transamination to indole pyruvic acid; and iv) the kynurenine pathway to kynurenine and its metabolites [8]. Pathways for induction of the tryptophan catabolite (TRYCAT) are commonly associated with the inflammation, and oxidative stress resulting from increased kynurenine levels. Kynurenine is an agonist of AhR; however, its metabolites kynurenic acid and quinolinic acid are AhR antagonists. Melatonin also acts as an antagonist to AhR [9]. Alpha-naphthoflavone (ANF) is a well-known synthetic antagonist of AhR, and it is widely used for the evaluation of toxicological and pharmacological AhR-mediated mechanisms [10]. Besides ligand-modulation, AhR can be activated in a ligand-independent manner by cyclic 3'5' adenosine monophosphate (CAMP) and protein kinase A (PKA) [11]. naphthoflavone
We recently reported a series of newly synthesized molecular hybrids bearing two highly promising pharmacophores, i.e., indole and aroylhydrazone moieties. For two of the substances, i.e., furan or thiophene substituted (corresponding respectively to the 3e- and 3f-substance) - Fig. 1, we have already discovered an acute anticonvulsant activity potentially mediated by the melatonin M1 receptors [12].

With the present study, we wanted to test 1) the potential chronic antiseizure activity; 2) the possible analgesic activity, and 3) the potential involvement of AhR. Substances with anticonvulsant activity are often tested for potential analgesic effects, thus trying to satisfy the patient's need for pain relief with better safety profile [13]. Electrical kindling is a model using subthreshold electrical stimulation of rodents, making them prone to develop spontaneous seizures. The pharmacological profile of the corneal kindling test in mice corresponds to partial epilepsy in humans and effectively identifies the anticonvulsant potential of active drugs under similar conditions, e.g., levetiracetam [14]. Additionally, we performed analgesic tests. We characterized the effect of indole-containing substances melatonin, $3 e$ and $3 f$, and alpha-naphthoflavone, a known AhR antagonist. The level of AhR and kynurenine in total brain homogenates were also evaluated.

\section{MATERIALS AND METHODS}

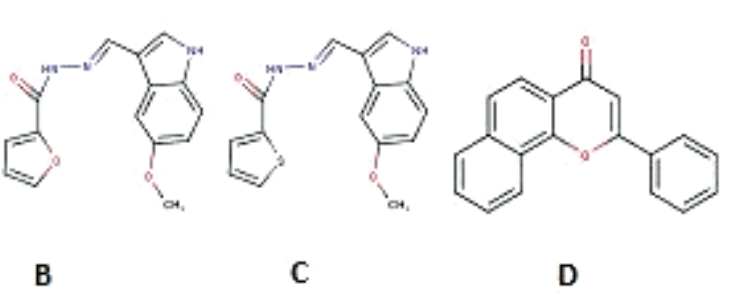

Fig. 1. Chemical structure of tested substances: A. Melatonin; B 3e; C 3f; D Alpha

\section{Drugs and Chemicals}

ANF and melatonin were purchased from Merck KGaA, Darmstadt, Germany. The two tested substances $3 e$ and $3 f$ were synthesized in the Chemistry Department of the Faculty of Pharmacy of the Medical University of Sofia. Their synthesis and spectral verifications were already reported elsewhere [12]. Three of these substances, i.e., melatonin, $3 \mathrm{e}$, and $3 \mathrm{f}$, have an indole structure. ANF was selected for this project as an AhR antagonist. All tested compounds were dissolved in saline and injected at various concentrations of 30, 100 and $300 \mathrm{mg} / \mathrm{kg}$. Each animal participated only once in an experiment. 


\section{Animals}

Adult male ICR mice (25-35 g) were obtained from the Vivarium of the Institute of Neurobiology of Bulgarian Academy of Sciences in Sofia. They were kept at a controlled temperature $\left(23 \pm 2^{\circ} \mathrm{C}\right)$ and relative humidity (50-70\%), at 12-12 hr light-dark cycle and free access to rodent chow and water. The animal models were tested under "the Principles of Laboratory Animal Care" formulated by the National Society for Medical Research and the Guide for the Care and Use of Laboratory Animals', Washington DC, National Academy Press. Approval N 246/08.10.2019 was received by the Bulgarian Food Safety Agency under the National Regulations.

\section{Induction of kindling by corneal electrical stimu- lation}

Electrical kindling was performed with ECT Unit 57800 (Ugo Basile, Gemonio, Italy). Mice were electrically stimulated twice daily with 3-second corneal stimulation with a current of $3 \mathrm{~mA}, 60 \mathrm{~Hz}$ for 14 days. After the 12 days of the 2-time kindling procedure, their seizure-prone activity reaches a plateau, and no further increases of severity can be expected. Seizure severity was ranked as follows: 1. Mild facial clonus and eye blinking. 2. Sever facial clonus, head nodding, chewing. 3. Unilateral or alternating forelimb clonus. 4. Bilateral forelimb clonus and rearing. 5. Bilateral forelimb clonus with rearing and falling. 6. Tonic hind-limb extension. Reaching stage 3 and more severity score was accepted as sufficient kindle.

After twice-daily corneal stimulation, mice typically reach stage 5 in 10-14 days. Twice daily stimulation was continued for each mouse until stage- 5 seizures were reached when it was assumed that the mouse had achieved complete kindling. Successfully kindled mice kept kindling throughout the day until all mice in the group have reached the state of "complete kindling." The substances were not tested in mice, who did not achieve complete kindling.

\section{Drug experiment}

The drug testing was performed in two sets of experiments. In the first set, drugs were applied to successfully kindled mice for testing their anticonvulsant activity.

Eight animals by the group were evaluated. All drug experiments were performed between 8:00 and 11:00 a.m. to minimize the circadian variance of the seizure susceptibility. Drugs were dissolved in saline and injected i.p. $30 \mathrm{~min}$ before electrostimulation. Several doses of the substances were evaluated.
To test the hypothesis about the involvement of AhR in the anticonvulsant effect of the studied substances, in some of the experiments, we injected the 100 $\mathrm{mg} / \mathrm{kg}$, i.p. ANF 30 minutes before the application of melatonin, 3e or 3f. The dose of ANF was based on the pharmacokinetic study performed by other authors [15]. Pre- and post-drug vehicle control experiments were done one day before and one day after the test. Each animal was used in the trial only once. Animal behavior was observed and assessed by two independent observers, unaware of the applied treatment.

For evaluation of $A h R$ and kynurenine in brain homogenates, we tested kindling-naïve mice. Data from other authors demonstrate that the corneal kindling test is related to an oxidative stress [16], and this factor is known to increase the kynurenine level. This part of our study aimed to evaluate AhR and kynurenine levels without the kindling procedure's interference. Mice were injected consecutively with the corresponding drug/dose group $(n=6)$ for 7 days. AhR and kynurenine were measured in brain homogenates.

\section{Hot plate test}

All experiments were performed after one week of adaptation, between 9.00 and 12.00 a.m. The mice were deprived from food the night before the experiment but had free access to water. Each animal was tested only once, 30 minutes after intraperitoneal injection of saline dissolved test compounds, at concentrations of 30,60 , or $100 \mathrm{mg} / \mathrm{kg}$. The control group was injected with the vehicle only. Acute thermal pain was elicited using a temperature of $47^{\circ}$ C. The test mice were placed in an open cylinder above the hotplate equipped with a thermostat and observed for two types of reactions - paw licking and jumping. The latency period for antinociceptive behavior was measured. This test was performed 30 minutes after the injection of the test substance.

\section{Formalin test}

Subdermal injection of $0.5 \%$ formalin was performed in the plantar region of the right mouse hind paw, thus triggering a respective 2 phase behavioral response. Immediately after injection, the mouse starts licking the paw for 5-10 minutes, corresponding to the acute phase of the test that is mediated by the activation of local C-fibers. A short latency period follows (usually less than 5 minutes) with no or minimal activity and a more prolonged inflammatory second phase (about 20-30 min) of licking, mediated mainly by inflammatory mechanisms and by the development of sensitization in the dorsal spine nerves. 
Animals were monitored to observe the effects of the test substances in both acute and inflammatory stages of persistent pain. Before administering the test substance, each mouse underwent a 15 -minute conditioning period in one of several standard Plexiglass cages. The mice $(n=8$ in the treatment group) were then monitored throughout the experiment. After the conditioning period, the test substance was administered intraperitoneally at different doses, and the mouse returned to the cage. Formalin was injected subcutaneously into the plantar part of the hind paw. After that, each animal was monitored by two independent investigators, and time in the first and second phases was counted in seconds.

\section{Brain homogenates}

The whole brains were harvested, weighed, and homogenized in ice with phosphate buffer saline (1:12 g: $\mathrm{ml})$ in an ultrasound homogenizer GX-10 (Bueno Biotech Ltd, Nanjing, China) with 1 second per impulse, $44 \mathrm{~W}$, time-off interval $1 \mathrm{sec}, 120$ cycles for 240 seconds. The homogenate was after that centrifuged at $0^{\circ} \mathrm{C}$ for $10 \mathrm{~min}$ in $15000 \mathrm{rpm}$. The supernatant was stored at $-80^{\circ} \mathrm{C}$ for further evaluation of AhR and kynurenine.

\section{AhR}

Mouse AhR ELISA kit was purchased from Wuhan Fine Biotech Co. Ltd, Wuhan, China. The principle of this kit is based on sandwich enzyme-linked immune-sorbent essay technology. The biotin-conjugated anti-AhR antibody was used as a detection antibody. Horseradish peroxidase (HRP)-streptavidin conjugate was added, and unbound conjugates were washed away with wash buffer. 3,3',5,5'-Tetramethylbenzidine (TMB) substrates were used to visualize HRP enzymatic reaction, read at $450 \mathrm{~nm}$ with ELx800 Biotech, Bad Friedrichshall, Germany.

\section{Kynurenine}

Mouse Kynurenine ELISA kit was purchased from ImmuSmal, Talence, France. The test is based on the competitive enzyme immunoassay method for the quantitative determination of L-kynurenine. In preparation for the examination, the samples were mixed with a derivatization reagent for the containing L-kynurenine. Subsequently, the derivatized sample and a polyclonal kynurenine antiserum in an ELISA plate coated with L-kynurenine derivative (tracer) were incubated. During the incubation, the target antigen competes with the tracer bound for binding the polyclonal antibody. In the second incubation step, a peroxidase-labeled secondary antibody was added, which binds to the L-kynurenine antibody. After a washing step to remove unbound components, the peroxidase substrate TMB was added. The enzyme reaction was visualized by HRP enzymatic reaction, changing the color from blue to yellow, which was read at $450 \mathrm{~nm}$ with ELx800 Biotech, Bad Friedrichshall, Germany.

\section{Statistics}

In cases where the data obtained showed normality of distribution and uniformity of variations, ANOVA was applied. In cases where these two conditions were not met, a non-parametric one-factor KruskalWallis analysis was used, followed by a Tukey test for group comparisons to identify statistically significant differences. In the analgesia test, a Dunnett test was applied to compare with the Wilcoxon control group. A repeated comparison test with the control was used to evaluate the severity of seizures (SS) of the exposed mice. A significance level of $p<$ 0.05 was accepted. Analyzes were performed with Sigma Plot 11.0.

\section{RESULTS}

The anticonvulsant activity of the indole derivatives in corneal kindling seizure model and lack of antagonistic effect by alpha naphthoflavone.

Mice were subjected to 3-second electrical stimulation at $3 \mathrm{~mA} 60 \mathrm{~Hz}$ and corneal electrodes to achieve five consecutive stage- 5 seizures (facial cloning and shaking of the head, progressing to a clone of the forelimbs, and finally rising and falling, accompanied by generalized clonic seizure). After twice-daily corneal stimulation, mice typically reach stage 5 in 10-14 days. Twice daily stimulation was continued for each mouse until the criterion of 5 consecutive stage- 5 seizures was reached when it was considered that complete kindling was achieved. The electrical treatment was continued until all mice in the group have reached the state of "complete kindling." The substances were not tested in mice, where full kindling was not achieved. The increase of the seizure severity during the kindling procedure is shown in Fig. 2.

The measurement of severity scores for the spontaneous seizures of kindled mice demonstrated effective suppression by melatonin (30,100 and $300 \mathrm{mg} /$ $\mathrm{kg}$ ), $3 \mathrm{e}$ (30 and $100 \mathrm{mg} / \mathrm{kg}$ ), and $3 \mathrm{f}$ (100 and $300 \mathrm{mg} /$ $\mathrm{kg})$ - Figure 3. The antagonist ANF in a dose of 100 $\mathrm{mg} / \mathrm{kg}$ did not abolish the seizure activity, indicating that $A h R$ is not involved in the mechanism of action of the studied substances. 


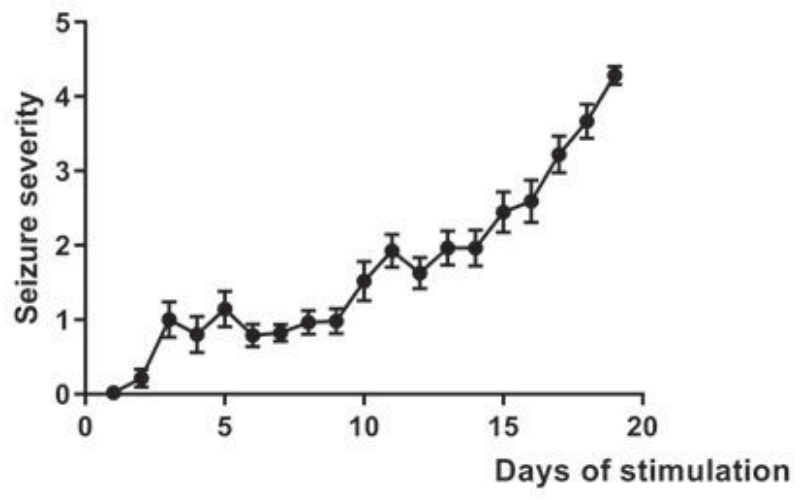

Fig. 2. The severity of epileptic seizures in the course of kindling. Data are presented through mean and error
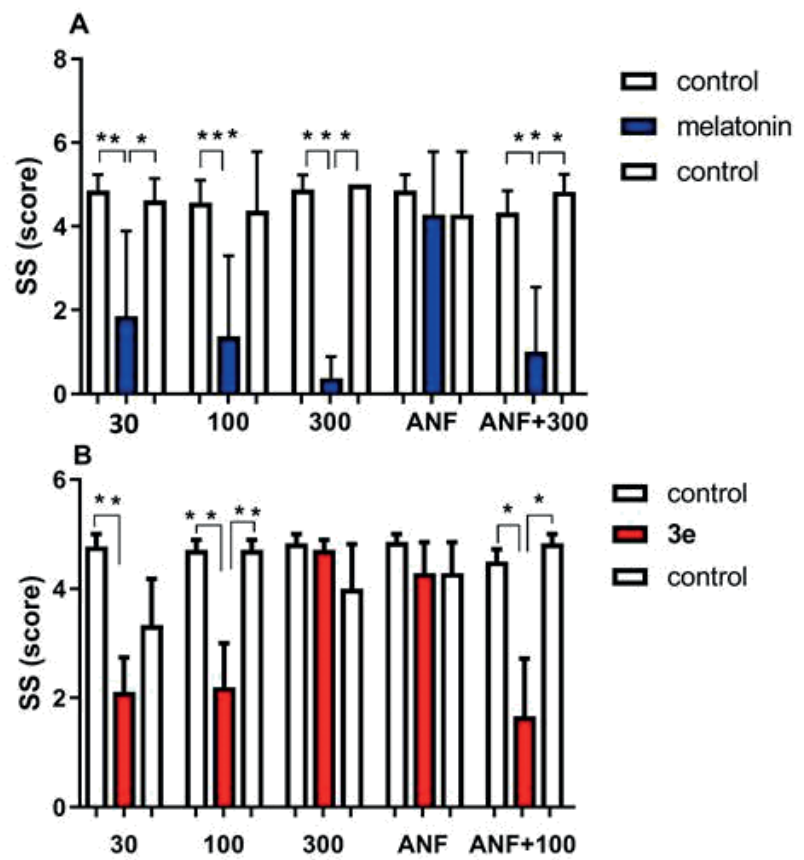

ㅁ control
ㅁ 3 e
control

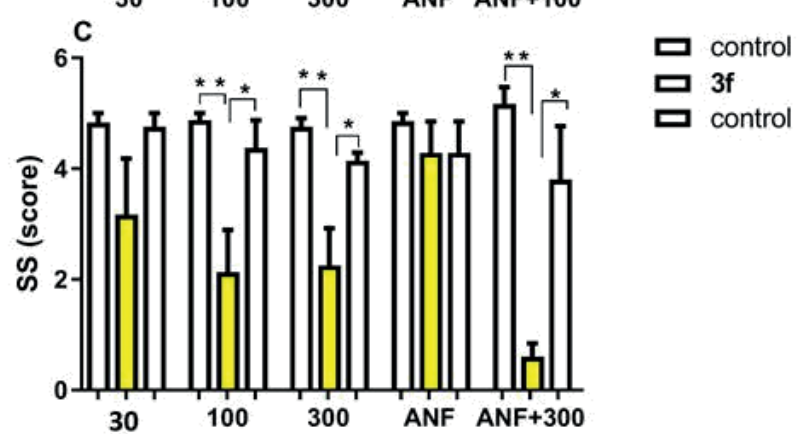

Fig. 3. Measurement of seizure severity with or without administration of $100 \mathrm{mg} / \mathrm{kg}$ ANF. The explanations are given in the text

\section{Hot plate test}

After assessing normality of distribution and equality of variance, a one-way ANOVA was applied, followed by a Dunnett test for comparison with the control group with $p<0.05$ acceptable level of significance. None of the treatment groups differed significantly from the control group - Figure 4.

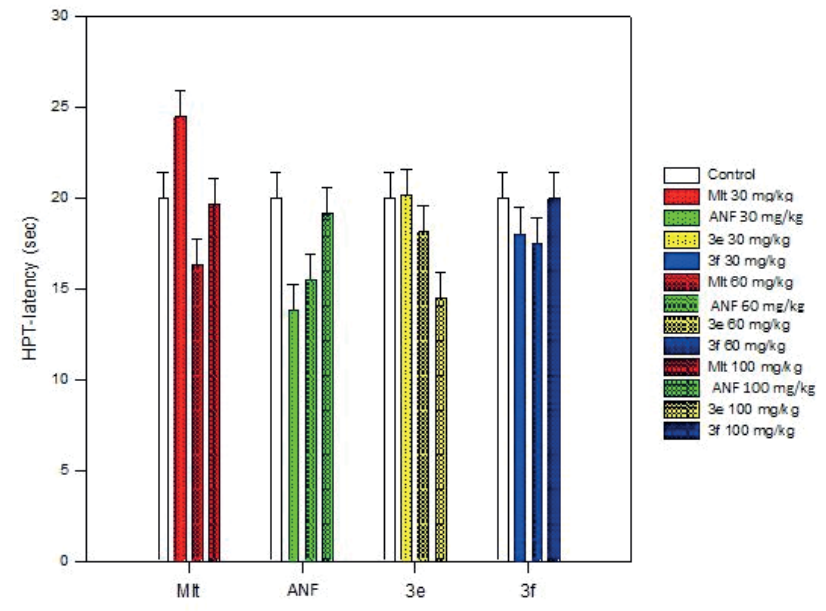

Fig. 4. Hot plate test - antinociceptive behavior latency (sec). $n=6$ in each group; $p>0.05$

\section{Formalin test - phase I (0-5 min)}

A two-factor ANOVA of the two independent variables (test substance, dose) of the latency period before antinociceptive behavior was applied - Figure 5. All effects were statistically significant at significance levels of 0.05 . The main effect of the test substance gave an $F$ ratio of $F(3,80)=13.772, p<0.001$, indicating a significant difference between the groups. The dose's main effect gave an $F$ ratio of $F(3,80)=$ $7.769, p<0.001$, indicating that the dose-effect was significant. The interaction effect was significant; $F$ $(9,80)=4.866, p<0.001$.

In the first phase of the formalin test, melatonin demonstrated the most potent analgetic effect in a dose-dependent manner. $3 \mathrm{e}$ at the lowest tested dose of $30 \mathrm{mg} / \mathrm{kg}$, even increased the antinociceptive behavior compared to melatonin.

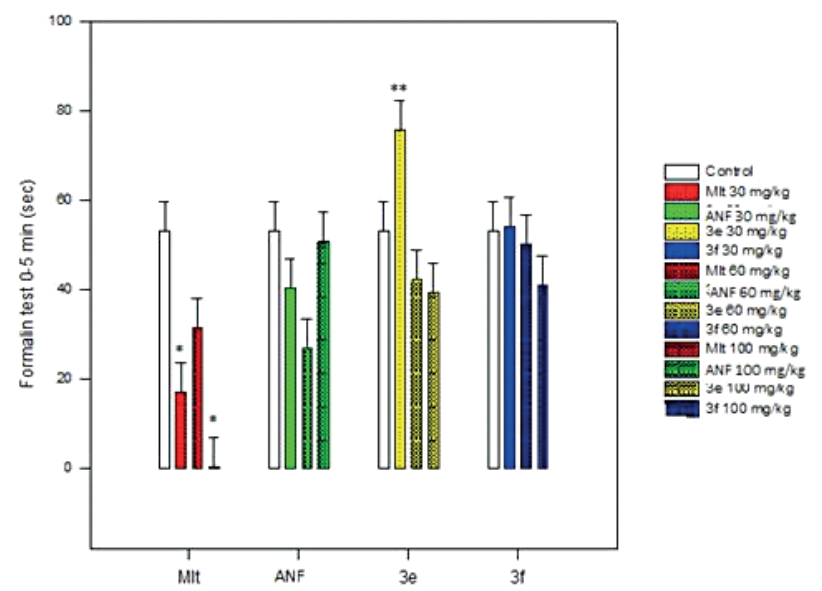

Fig. 5. Results of the first phase of the formalin test. The explanations are given in the text

\section{Formalin test phase II}

A two-factor ANOVA was used for both factors administered substances and doses. Statistically, 
a significant interaction was identified between two factors, $F(9,80)=5,505$. $P<0.001$ Melatonin, both substances $3 e$ and $3 f$ showed statistically significant lower values (analgesic effect) than controls ( $p<$ 0.05). ANF did not show analgesic effect - Figure 6.

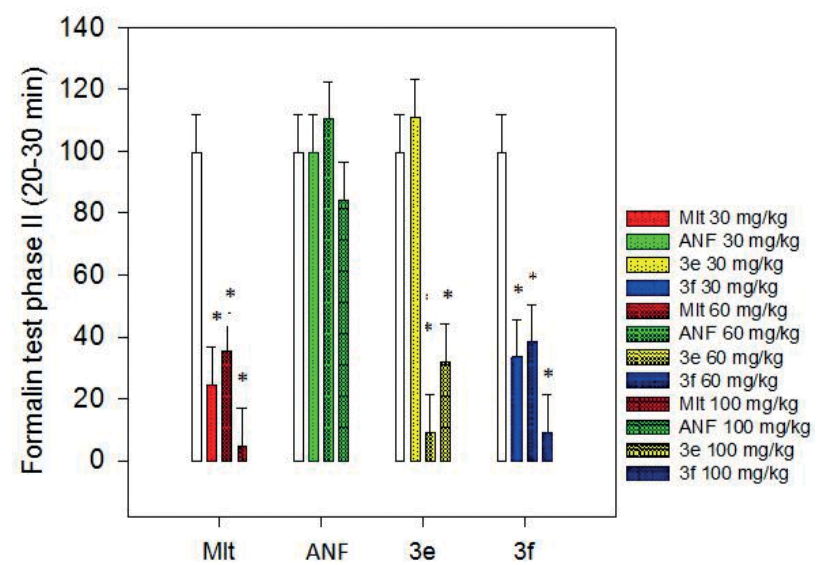

Fig. 6. Results of the second phase of the formalin test. The explanations are given in the text

\section{AhR levels in brain homogenates}

After 7-day treatment with melatonin or $3 \mathrm{f}$, a dosedependent increase in the level of AhR in the brain homogenates was detected. After applying the
Kruskal-Wallis test for melatonin, $\mathrm{H}=9.266$ with 3 degrees of freedom, $p=0.026$, and for substance $3 f$ and $H=10.325$ with 3 degrees of freedom, $p=0.016$, Figure 7.

Higher AhR levels were recorded in the $300 \mathrm{mg} / \mathrm{kg}$ dose group in melatonin treated mice, and this was statistically significant (higher than the $30 \mathrm{mg} / \mathrm{kg}$ treatment group $(p<0.05)$ ). For substance $3 f$, AhR levels in the $300 \mathrm{mg} / \mathrm{kg}$ treated group also showed statistically significant higher AhR levels than the control and $30 \mathrm{mg} / \mathrm{kg}$ treated groups ( $<<0.05)$.

\section{Kynurenine level in brain homogenates}

In determining kynurenine levels, significant changes were observed after administering different doses of melatonin and substance $3 \mathrm{f}$. In contrast, no significant differences were observed after administering different doses of ANF and substance 3e. After one way administration of different doses melatonin ANOVA, $F(3.20)=7.171, p=0.002$ was found. Significantly higher levels were found at doses of $100 \mathrm{mg} / \mathrm{kg} p<$ 0.05 . Regarding substance $3 \mathrm{f}$, the administration of ANOVA also showed that different treatment doses had a significant effect differences $-F(3,20)=3,643$, $p=0.030$. Significantly higher levels were found at doses of $100 \mathrm{mg} / \mathrm{kg} \mathrm{p}<0.05-$ Figure 8 .
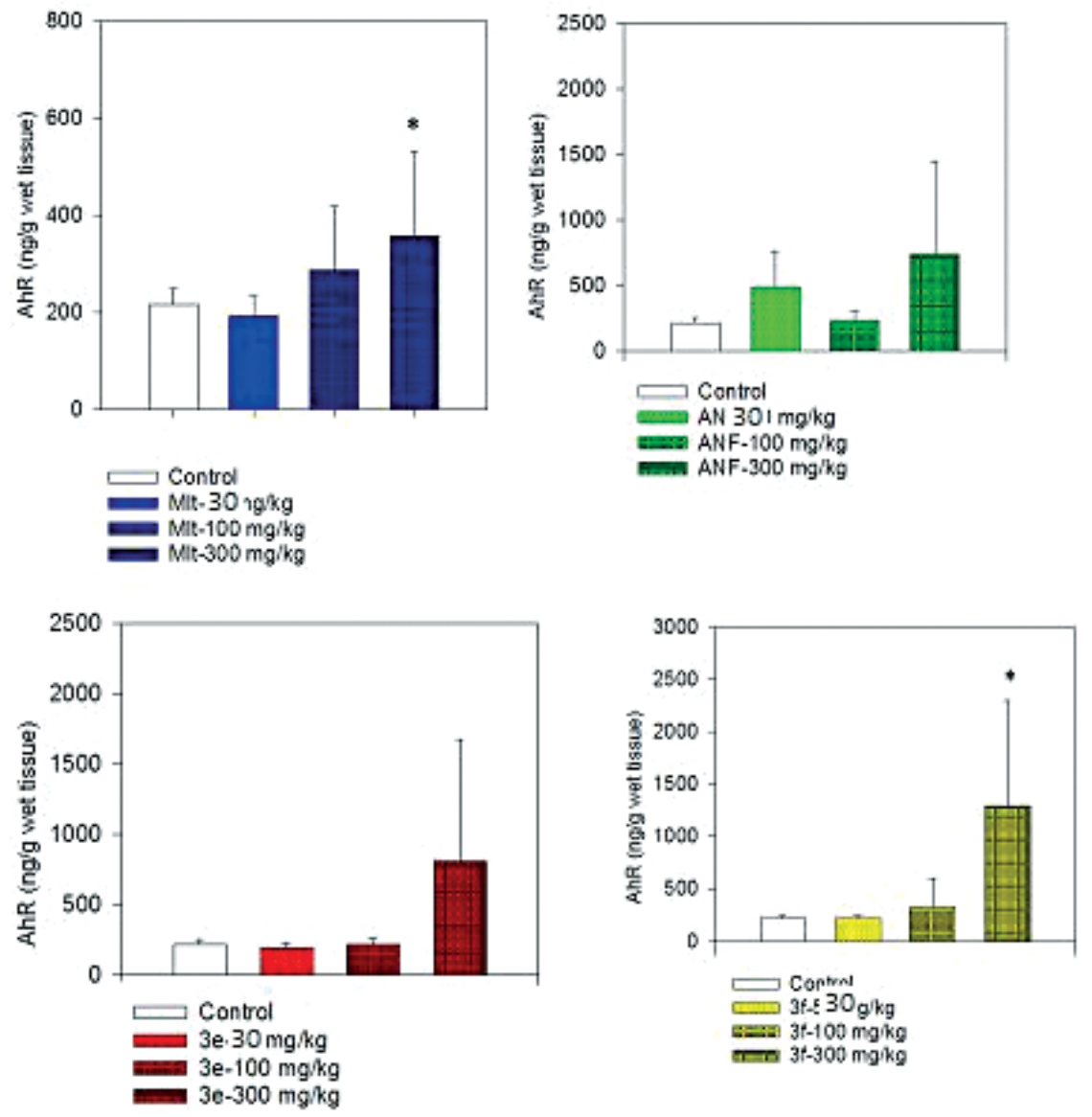

Fig. 7. AhR levels in brain homogenates after seven days of treatment with melatonin, ANF, $3 e$ or $3 f .{ }^{*} P<0.05$ 

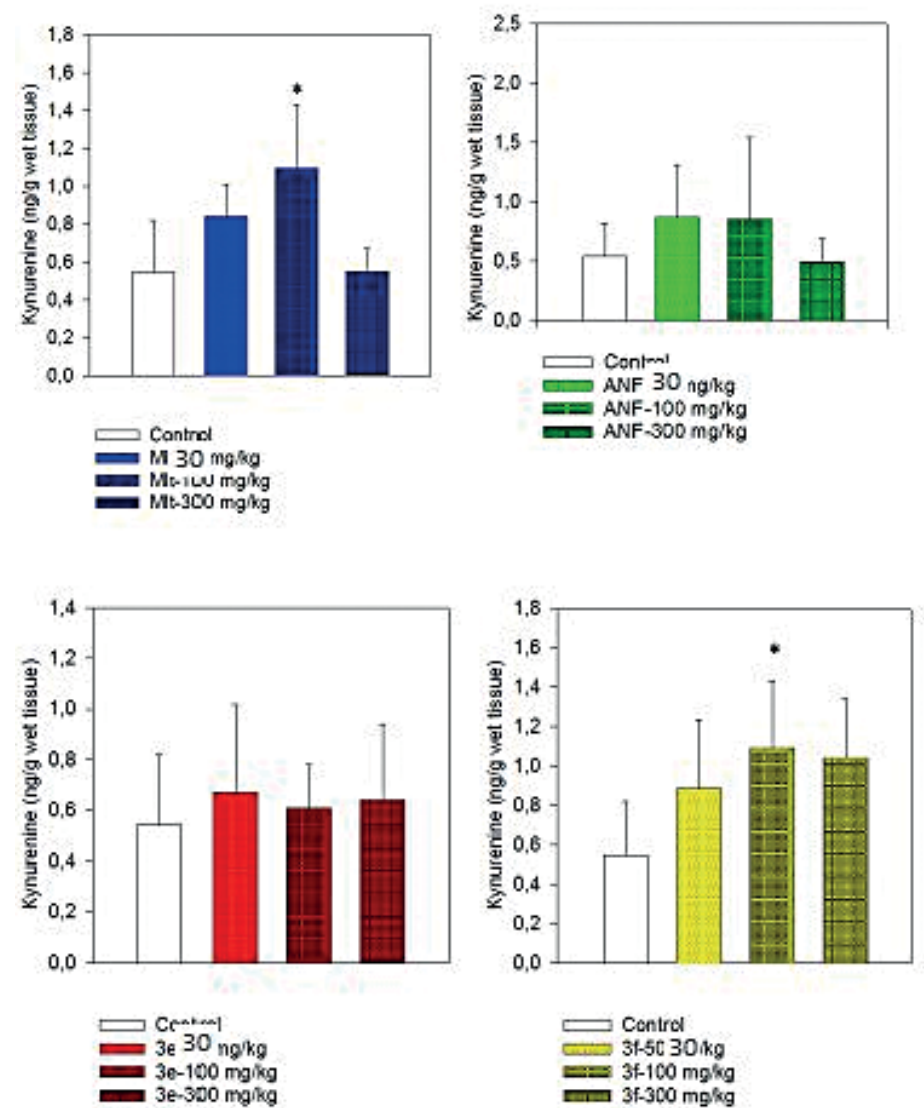

$$
\begin{aligned}
& \square \text { Control } \\
& 31-5030 \mathrm{~kg} \\
& =3 f-100 \mathrm{mg} / \mathrm{kg} \\
& =3 f-300 \mathrm{mg} / \mathrm{kg}
\end{aligned}
$$

Fig. 8. Kynurenine levels in brain homogenates after a 7-day course of treatment with melatonin, ANF, 3e or $3 f .{ }^{*} P<0.05$

\section{DISCUSSION}

We conducted a neuropharmacological screening of two indole derivatives with an aroylhydrazone moiety, comparing them with melatonin (indole derivative) and alfa-naphthoflavone (AhR). The tested substances (melatonin, ANF, 3e, and 3f) showed a lack of neurological and muscular toxicity with the rotarod test. For the compounds $3 e$ and $3 f$, we found antiepileptic activity comparable to one of melatonin after kindling test administration and some analgesic activity, albeit weaker, than that of melatonin in equivalent doses. Although melatonin and substance $3 \mathrm{f}$ showed dosedependent increases in AhR and kynurenine levels in brain homogenates, our results do not support our initial hypothesis for AhR involvement in the analgesic or antiepileptic mechanisms of test substances. Further studies are needed to evaluate the effects of AhR and kynurenine in the neuropharmacological activity of the indole derivatives, with or without the aroylhydrazone moiety.

In recent years, many research teams have implemented targeted syntheses and development of melatonin analogs with potential pharmacological activity similar to endogenous melatonin and with advantag- es associated with prolonged action. Scientific studies focus on the development of melatonin analogs for various diseases, such as depression, Alzheimer's disease, anxiety, circadian rhythm disorders, and insomnia [17].

Clinical and experimental data in several animal models, including the temporal lobe epilepsy model, confirm the anticonvulsant role of the pineal hormone melatonin [18]. Its advantages are good safety profile (being an endogenous compound), potent antioxidant activity, contribution to degeneration protection of neurons against the mutagenic and carcinogenic effect of oxidants, and above all, its essential biological function to synchronize natural rhythms. Melatonin alleviates sleep disorders of various origins in both children [19] and adults with epilepsy [20]. In most cases, it has been found that improving sleep quality is associated with a decrease in seizure activity in young patients with resistant epilepsy and patients receiving antiepileptic treatment [21]. While some of the routinely used anticonvulsants such as lamotrigine, levetiracetam, valproate, gabapentin do not show improvements in the architectonics of sleep, other drugs such as benzodiazepines, barbiturates and phenytoin impair the quality of sleep in epilepsy patients [22].

Substances with anticonvulsant activity are often tested for analgesic effects, trying to find alternatives to satisfy patients' needs for pain relief with favorable safety profile [23]. Melatonin is a hormone with several neurophysiological and antioxidant properties that have been increasingly studied recently. In supra-physiological doses, it is authorized for use as a medicine in cases of insomnia associated with disturbed circadian rhythms. Due to its favorable safety profile, it is widely used as a nutritional supplement.

Numerous preclinical studies indicate the analgesic potential of melatonin, administered alone or in addition to well-established analgesics, where it shows synergism with analgesia [24]. There are promising, but still limited data on the administration of melatonin in humans to respond to pain [25].

The AhR is most widely expressed in the human placenta, followed by the lungs, heart, pancreas, and liver. It was also detected in the kidney, the CNS, and the skeletal muscles [26]. In the brain, AhR is thought to mediate the host-microbiome interaction with a particular implication in the neurodevelopment [27]. The brain expression of AhR shows low region specificity [28]; therefore, we evaluated the concentration of the AhR in whole brain homogenates. 
Other authors investigated the dysregulation of the AhR as a cause for tumorigenesis, autism spectrum disorders, multiple sclerosis, depression, leaky gut and blood-brain barrier, stroke, epilepsy, and the universal mechanism seems to be suboptimal mitochondrial functioning. One plausible cause of the mitochondrial dysfunction could be an increasing $\mathrm{N}$ acethylserotonin/melatonin ratio due to AhR activation [29]. As it is already known, all body cells can produce melatonin, a circadian regulator, and powerful antioxidant, which synthesis occurs in mitochondria [30]. Its synthetic pathway can be diverted to the kynurenine pathway with the production of kynurenic acid and quinolinic acid, substances with the opposite to melatonin effects in terms of neuroprotection and neuronal overexcitation. One of the significant consequences of the neurovascular activation by $\mathrm{AhR}$ is an increasing $\mathrm{N}$-acethylserotonin/melatonin ratio and modulating mitochondrial function [31].

Besides ligand-modulation, AhR can be activated in a ligand-independent manner by cyclic 3'5' adenosine monophosphate (cAMP) and protein kinase $A$ (PKA) [32]. PKA is a ubiquitously expressed member of the serine-threonine kinases implicated in multiple cellular processes, including neuronal excitability [33]. As reported by other authors, repeated injection of an initially sub convulsive dose of cAMP into the rat amygdala produced progressive seizure development, like that of electrical kindling [34]. We hypothesized that the application of an inhibitor of AhR could, after that, suppress such activity. However, our results did not support this hypothesis.

The effect of melatonin on circadian rhythms is very well described. However, we do not know if our two tested substances, $3 \mathrm{e}$ and $3 \mathrm{f}$, being $\mathrm{M} 1$ agonists, according to our previous work, also interfere with the circadian rhythms. We do know, however, from other authors that both AhR and ARNT proteins display diurnal changes in several evaluated tissues [35]. Although the AhR has no effect on the circadian rhythms in the absence of exogenous agonists, upon activation, AhR may affect the ability of the endogen timekeeping system to adjust the canonical clock genes to alterations [36]. AhR activation in mice and hamsters alters rhythms of feeding and activity [37], shifts the surge of corticosterone, and reduces the level of prolactin [38] and melatonin [39].

\section{CONCLUSION}

In this study we have confirmed the analgesic and anticonvulsant potential of melatonin. In addition, we performed neuropharmacological studies of two substances, melatonin analogs with the aroylhydrazone moiety, and reported objectifying results with respect to the activities tested. The theoretical contribution is with regard to the reporting of data on AhR and kynurenine levels in brain homogenates after seven days of treatment with the test substances. These data could be used in future studies regarding AhR and its relationship to the kynurenine metabolic pathway. Indoles derived from an exogenous diet, such as tryptophan, are a major source of endogenous AhR ligand precursors. It is known from studies by other authors that kynurenine itself is an AhR agonist; while its metabolites kynurenic acid and quinoline acid and also melatonin are AhR antagonists.

Acknowledgment: The authors received financial support for the research from Medical University - Sofia (grant D-62/2019) and National Science Fund of Bulgaria (grant DN 13/16 21.12.2017).

\section{REFERENCES}

1. Stevens EA, Mezrich JD, Bradfield CA. The aryl hydrocarbon receptor: a perspective on potential roles in the immune system. Immunology. 2009;127(3):299-311.

2. Salisbury TB, Tomblin JK, Primerano DA, et al. Endogenous aryl hydrocarbon receptor promotes basal and inducible expression of tumor necrosis factor target genes in MCF-7 cancer cells. Biochem Pharmacol. 2014;91(3):390-399.

3. Hernández-Ochoa I, Karman BN, Flaws JA. The role of the aryl hydrocarbon receptor in the female reproductive system. Biochem Pharmacol. 2009;77(4):547-559.

4. Luecke-Johansson S, Gralla M, Rundqvist H, et al. AMolecular Mechanism To Switch the Aryl Hydrocarbon Receptor from a Transcription Factor to an E3 Ubiquitin Ligase. Mol Cell Biol. 2017;37(13):e00630-16.

5. Murray IA, Perdew GH. Ligand activation of the Ah receptor contributes to gastrointestinal homeostasis. Curr Opin Toxicol. 2017;2:15-23.

6. Busbee PB, Rouse M, Nagarkatti M, Nagarkatti PS. Use of natural AhR ligands as potential therapeutic modalities against inflammatory disorders. Nutr Rev. 2013;71(6):353-369.

7. Badawy AA. Tryptophan Metabolism: A Versatile Area Providing Multiple Targets for Pharmacological Intervention. Egypt J Basic Clin Pharmacol. 2019;9:10.32527/2019/101415.

8. Anderson G, Maes M. Interactions of Tryptophan and Its Catabolites With Melatonin and the Alpha 7 Nicotinic Receptor in Central Nervous System and Psychiatric Disorders: Role of the Aryl Hydrocarbon Receptor and Direct Mitochondria Regulation. Int J Tryptophan Res. 2017;10:1178646917691738.

9. Yamamoto J, Ihara K, Nakayama H, et al. Characteristic expression of aryl hydrocarbon receptor repressor gene in human tissues: organ-specific distribution and variable induction patterns in mononuclear cells. Life Sci. 2004;74(8):10391049.

10. Soshilov AA, Denison MS. Ligand promiscuity of aryl hydrocarbon receptor agonists and antagonists revealed by sitedirected mutagenesis. Mol Cell Biol. 2014;34(9):1707-1719. 
11. Tischkau SA. Mechanisms of circadian clock interactions with aryl hydrocarbon receptor signalling. Eur J Neurosci. 2020;51(1):379-395.

12. Tchekalarova J, Angelova VT, Todorova N, Andreeva-Gateva $P$, Rangelov M. Evaluation of the anticonvulsant effect of novel melatonin derivatives in the intravenous pentylenetetrazol seizure test in mice. Eur J Pharmacol. 2019;863:172684.

13. Arsene AL, Mitrea N, Cristea A, et al. Experimental research on mice regarding the implication of melatonin in pain management. Farmacia 2009;57:223-8.

14. Rowley NM, White HS. Comparative anticonvulsant efficacy in the corneal kindled mouse model of partial epilepsy: Correlation with other seizure and epilepsy models. Epilepsy Res. 2010;92(2-3):163-169.

15. Jilek JL, Tian Y, Yu AM. Effects of MicroRNA-34a on the Pharmacokinetics of Cytochrome P450 Probe Drugs in Mice. Drug Metab Dispos. 2017;45(5):512-522.

16. Koshal P, Kumar P. Effect of Liraglutide on Corneal Kindling Epilepsy Induced Depression and Cognitive Impairment in Mice. Neurochem Res. 2016;41(7):1741-1750.

17. Ying SW, Rusak B, Delagrange P, Mocaer E, Renard P, Guardiola-Lemaitre B. Melatonin analogues as agonists and antagonists in the circadian system and other brain areas. Eur $\mathrm{J}$ Pharmacol. 1996;296(1):33-42.

18. Mohammadi F, Shakiba S, Mehrzadi S, Afshari K, Rahimnia AH, Dehpour AR. Anticonvulsant effect of melatonin through ATP-sensitive channels in mice. Fundam Clin Pharmacol. 2020;34(1):148-155.

19. Peled N, Shorer Z, Peled E, Pillar G. Melatonin effect on seizures in children with severe neurologic deficit disorders. Epilepsia. 2001;42(9):1208-1210.

20. Reddy DS, Chuang SH, Hunn D, Crepeau AZ, Maganti R. Neuroendocrine aspects of improving sleep in epilepsy. Epilepsy Res. 2018;147:32-41.

21. Manni R, De Icco R, Cremascoli R, et al. Circadian phase typing in idiopathic generalized epilepsy: Dim light melatonin onset and patterns of melatonin secretion-Semicurve findings in adult patients. Epilepsy Behav. 2016;61:132-137.

22. Roshan S, Puri V, Chaudhry $\mathrm{N}$ et al. Sleep abnormalities in juvenile myoclonic epilepsy-A sleep questionnaire and polysomnography based study. Seizure. 2017;50:194-201.

23. Arsene AL, Mitrea N, Cristea A, et al. Experimental research on mice regarding the implication of melatonin in pain management. Farmacia 2009;57:223-8.

24. Kuthati Y, Lin SH, Chen IJ, Wong CS. Melatonin and their analogs as a potential use in the management of Neuropathic pain. J Formos Med Assoc. 2019;118(8):1177-1186.

25. Zhu C, Xu Y, Duan Y, et al. Exogenous melatonin in the treatment of pain: a systematic review and meta-analysis. Oncotarget. 2017;8(59):100582-100592.

26. Uhlén M, Fagerberg L, Hallström BM, et al. Tissue-based map of the human proteome. Science 2015 347(6220):1260419.
27. Angelova VT, Rangelov $M$, Todorova $N$, et al. Discovery of novel indole-based aroylhydrazones as anticonvulsants: Pharmacophore-based design. Bioorg Chem. 2019;90:103028.

28. Anderson G, Reiter RJ. Glioblastoma: Role of Mitochondria $\mathrm{N}$-acetylserotonin/Melatonin Ratio in Mediating Effects of miR-451 and Aryl Hydrocarbon Receptor and in Coordinating Wider Biochemical Changes. International Journal of Tryptophan Research: IJTR. 2019 ;12:1178646919855942.

29. Anderson G, Maes M. Gut Dysbiosis Dysregulates Central and Systemic Homeostasis via Suboptimal Mitochondrial Function: Assessment, Treatment and Classification Implications [published online ahead of print, 2020 Jan 30]. Curr Top Med Chem. 2020;10.2174/15680266206662001 31094445.

30. Tan DX, Manchester LC, Qin L, Reiter RJ. Melatonin: A Mitochondrial Targeting Molecule Involving Mitochondrial Protection and Dynamics. Int J Mol Sci. 2016;17(12):2124.

31. Lee HU, McPherson ZE, Tan B, Korecka A, Pettersson S. Host-microbiome interactions: the aryl hydrocarbon receptor and the central nervous system. J Mol Med (Berl). 2017;95(1):29-39.

32. Tischkau SA. Mechanisms of circadian clock interactions with aryl hydrocarbon receptor signalling. Eur J Neurosci. 2020;51(1):379-395.

33. Bracey JM, Kurz JE, Low B, Churn SB. Prolonged seizure activity leads to increased Protein Kinase $A$ activation in the rat pilocarpine model of status epilepticus. Brain Res. 2009;1283:167-176.

34. Yokoyama N, Mori N, Kumashiro H. Chemical kindling induced by CAMP and transfer to electrical kindling. Brain Res. 1989;492(1-2):158-162.

35. Richardson VM, Santostefano MJ, Birnbaum LS. Daily cycle of bHLH-PAS proteins, Ah receptor and Arnt, in multiple tissues of female Sprague-Dawley rats. Biochem Biophys Res Commun. 1998;252(1):225-231.

36. Shimba S, Watabe Y. Crosstalk between the AHR signaling pathway and circadian rhythm. Biochem Pharmacol. 2009;77(4):560-565.

37. Jaeger C, Tischkau SA. Role of Aryl Hydrocarbon Receptor in Circadian Clock Disruption and Metabolic Dysfunction. Environ Health Insights. 2016;10:133-141.

38. Jones MK, Weisenburger WP, Sipes IG, Russell DH. Circadian alterations in prolactin, corticosterone, and thyroid hormone levels and down-regulation of prolactin receptor activity by $2,3,7,8$-tetrachlorodibenzo-p-dioxin. Toxicol Appl Pharmacol. 1987;87(2):337-350.

39. Linden J, Pohjanvirta R, Rahko T, Tuomisto J. TCDD decreases rapidly and persistently serum melatonin concentration without morphologically affecting the pineal gland in TCDD-resistant Han/Wistar rats. Pharmacol Toxicol. 1991;69(6):427-432. 